\title{
Magnetic Resonance Guided Radiotherapy for Cardiac Lymphomas: Report of a Case and New Perspectives
}

\author{
Angela Romano ${ }^{1}$, Annarosa Cuccaro ${ }^{1}$, Eugenia De Marco², Luca Boldrini ${ }^{1}$, \\ Mauro lafrancesco ${ }^{2}$, Francesco D'Alò ${ }^{1}$, Silvia Chiesa1, Ciro Mazzarella1, \\ Stefan Hohaus ${ }^{1}$, Mario Balducci ${ }^{1}$ \\ ${ }_{1}^{1}$ Dipartimento di Diagnostica per immagini, Radioterapia oncologica ed Ematologia - Fondazione \\ Policlinico Universitario "A. Gemelli" IRCCS, Rome, Italy \\ ${ }^{2}$ Dipartimento di Scienze Cardiovascolari- Fondazione Policlinico Universitario "A. Gemelli" IRCCS, \\ Rome, Italy
}

Corresponding Author: Luca Boldrini; email: luca.boldrini@policlinicogemelli.it

\section{Abstract}

Cardiac lymphomas are extremely rare and have poor prognoses. Currently, there are no established guidelines for treatment and the main approaches include surgery, chemotherapy ( $\mathrm{CHT}$ ), possibly combined with radiotherapy (RT), and autologous stem cell transplantation (ASCT). RT's role is controversial and is not considered a standard approach. We describe the case of a patient diagnosed with cardiac lymphoblastic Bcell lymphoma/ acute Lymphoblastic B-cell leukemia and successfully treated with a multimodality approach, including CHT, RT, and ASCT. In particular, the patient was referred to magnetic resonance-guided RT (MRgRT), currently the most advanced available technology in the RT field, which maximizes therapeutic efficacy by exploiting the best soft-tissue resolution of the magnetic resonance images and efficient gating protocols during treatment delivery.

Keywords: cardiac lymphoma, MR guided radiotherapy, gating, MRgRT

\section{Introduction}

Primary cardiac tumors are an infrequent clinical entity, with an average incidence of $0.02 \%$ in autopsy series. Of these,
$75 \%$ are benign. Among malignant tumors, the most frequent are sarcomas and lymphomas. Primary cardiac lymphoma (PCL) constitutes about $1.3 \%$ of primary cardiac neoplasms, $80 \%$ of which are diffuse large 
cell B-cell lymphomas (DLBCL). PCL has a poor prognosis, with an overall median survival of 12 months (1-3). The median age of patients is 63 years and PCL more often involves the right heart (both atrium and ventricle) (4). In most cases, it presents with congestive heart failure due to blood flow obstruction secondary to intracardiac mass growth, but often only with non-specific symptoms, such as dyspnea, chest pain, and general malaise. It may also present as an oncological emergency. The rapid clinical evolution related to advanced organ infiltration in these cases causes cardiac arrythmias and atrioventricular blocks potentially leading to sudden death (1). PCL treatment includes various therapeutic modalities, including $\mathrm{CHT}$, eventually combined with RT, surgery, and even autologous stem cell transplantation (ASCT) (2). The evolution of diagnostic imaging techniques and the introduction of Rituximab in $\mathrm{CHT}$ regimens has led to an improved prognosis in PCL patients. Also, modern RT can play an innovative role in association with CHT protocols (5). Recent advancements in RT introduced magnetic MRgRT, as the most advanced RT delivery technology currently available. MRgRT has several clinical applications, as it combines enhanced soft-tissue visualization offered by the onboard MRI, and the direct gating options for motion management purposes. These benefits result in safer irradiation, ensuring that healthy organs are spared efficiently (6). We describe the first case of a patient with intracardiac lymphoblastic Bcell lymphoma/ acute Lymphoblastic B-cell leukemia managed multidisciplinary, and successfully treated with $\mathrm{CHT}, \mathrm{MRgRT}$, and ASCT.

\section{Case presentation/ History Presentation}

A 60-year-old woman with no past medical history was admitted to the emergency department in March 2019 for progressive worsening of dyspnea and asthenia over a three months period. Results from blood tests were within normal ranges, but the ECG showed atrial fibrillation with a high ventricular response. Lower limbs edemas were present, and the patient was diagnosed with heart failure and referred to imaging studies.

A chest CT scan excluded pulmonary embolism and showed a large right atrial lesion. A transthoracic echocardiogram (TTE) and a trans-esophageal echocardiogram (TEE) identified a solid cardiac lesion and a residual ejection fraction (EF) of $42 \%$.
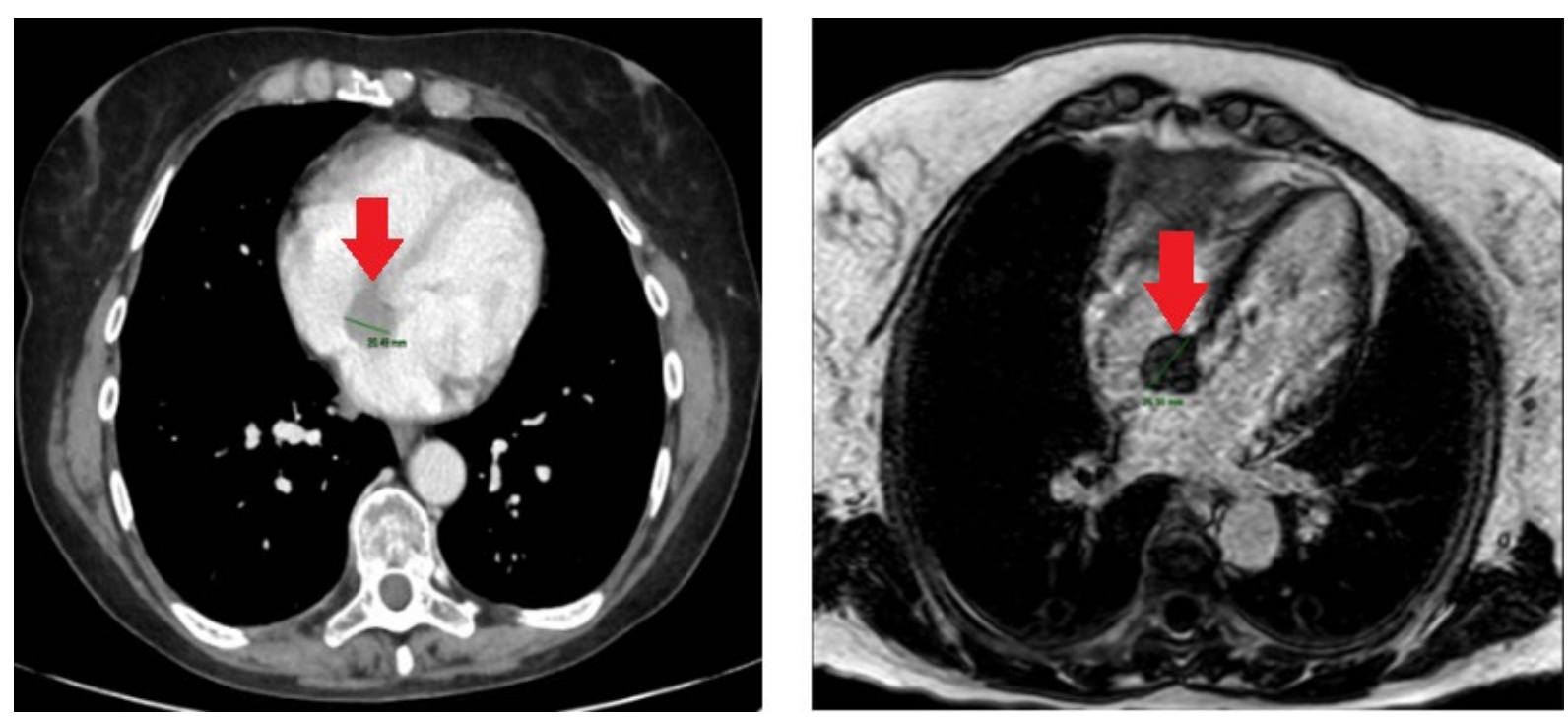

Figure 1. CT (left) and cardiac MRI (right) staging images. The lesion is indicated by red arrows. 
The lesion was later confirmed by a cardiac 1.5 T magnetic resonance (MR) (GE Signa Excite, Medical Systems), and measured $48(d A P) \times 63(d L L) \times 53(d C C) \mathrm{mm}$. The lesion infiltrated the interatrial septum, the superior venous sinus, and the right atrial roof, surrounding the aortic root almost circumferentially. This finding raised the diagnosis of primary cardiac lymphoma (PCL). Figure 1 shows diagnostic imaging performed for staging.

A biopsy of the lesion was performed in April 2019. Pathologic examination showed fragments of fibro-adipose tissue with a solid structure consisting of small, monomorphic cells, diffusely positive for LCA, TdT, PAX5, and CD10, and negative for CD3, CK $A E 1 / E 3$, and vimentin on immunohistochemistry. Ki67 proliferative index was $90 \%$. These pathologic findings were compatible with lymphoblastic B-cell lymphoma/ acute Lymphoblastic B-cell leukemia and correlated well with the imaging findings.

The patient underwent disease staging, with bone marrow aspiration and biopsy, and cytogenetic and molecular biology investigations that showed no signs of lymphomatous involvement. She was referred for treatment with chemotherapy.

The patient underwent first-line chemotherapy $(\mathrm{CHT})$ according to the GMALLALL/NHL 2002 scheme (including vincristine, methotrexate, cyclophosphamide, and doxorubicin), and she completed A1, B1, A2, B2, and A3 cycles from May 2019 to September 2019. Disease re-evaluation was then performed with TTE and total body CT in September 2019, documenting stable disease according to RECIST criteria. Consequently, the B3 CHT cycle was administered in October 2019.

CHT course has been complicated by G3 thrombocytopenia, G3 anemia, and G3 neutropenia (CTCAE 4.0 scale).

The restaging ${ }^{18} \mathrm{~F}-\mathrm{FDG}$ PET-CT scan performed in November 2019 showed intense uptake (SUVmax 12) at the level of the interatrial septum known hypodense tissue, with extension to the aortic root, consistent with persistent/recurrent disease. TTE, cardiac MRI, and total body CT were also performed in January 2020, confirming disease persistence/recurrence.

The case was discussed by a multidisciplinary team of hematologists, cardiologists, and radiation oncologists. The decision was to perform inpatient radiotherapy (RT) treatment of the intracardiac lesion, due to increased cardiac risk. Regular TTE and weekly troponin tests were also done.

The patient was then referred to Magnetic Resonance guided Radiotherapy (MRgRT) with a total dose of $30.6 \mathrm{~Gy}$ in 17 consecutive fractions on a 0.35 T $6 \mathrm{MV}$ hybrid MR-Linac (MRIdian, ViewRay Inc., USA). This technology was chosen as it takes advantage of MR imaging and direct gating approaches.

A double source of motion had to be managed in this case, with the therapy volumes position being prone to breathing, and heartbeat related spontaneous movements. The treatment was carried out in deep inspiration breath hold (DIBH) based on these evaluations.

The segmentation of both target volumes and organs at risk (OARs) was performed using the 25-second true fast imaging (TRUFI), a type of balanced steadystate free precession (bSSFP) sequence, yielding aT2/T1-weighted contrast, $\mathrm{DIBH}$ scans (see figure 2) co-registered with diagnostic imaging available (contrast-enhanced CT and cardiac MR scans), with the assistance of a radiologist specialized in cardiac neoplasms. The cardiac MR sequences used were both T1- and T2-weighted, and contrast-enhanced, to help discriminate between pathological tissue and areas of inflammatory or healthy tissue.

During each RT fraction, the patient could see through a mirror the movement of the target on the online cine MRI acquired on a sagittal plane. Dose delivery was automatically turned off when the target volume moved outside of the boundary region set 
prior to delivery. Specifically, a sagittal slice of a volumetric MRI is displayed. It is possible to set the gating structure on this plane (e.g. the target), with the percentage of the target allowed outside the gating structure for the beam to turn off (direct gating).

The patient can actively participate in the treatment by modulating the breathing cycles to put the target in the proper position and thereby to increase gating compliance, thanks to a dedicated visual feedback system through which the cine-MRI is projected on an MR compatible monitor positioned on the vault's wall.

The patient started treatment in February 2020 , and RT sessions were well tole- rated with no interruptions. After $10 \mathrm{RT}$ fractions, the patient developed two episodes of paroxystic supraventricular tachycardia, which were managed pharmacologically (Verapamil $2.5 \mathrm{mg} \mathrm{iv}$ ), restoring sinus rhythm.

The subsequent PET-CT scan performed four weeks after the end of RT demonstrated a complete response of the cardiac lesions. However, a new uptake area was observed in the left tibia, and a biopsy later confirmed metastatic disease that was treated with $3 D$ conformal radiotherapy (36 Gy in 18 fractions).

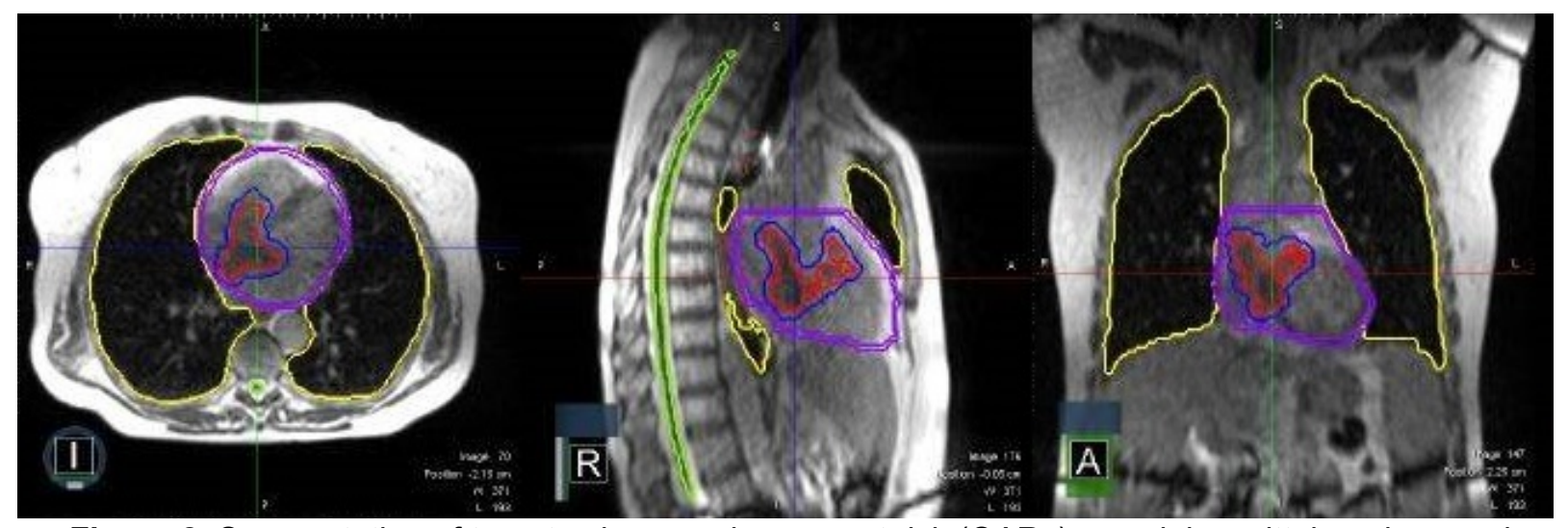

Figure 2. Segmentation of target volume and organs at risk (OARs) on axial, sagittal, and coronal TRUFI simulation scans. The gross tumor volume (GTV) is highlighted in red.

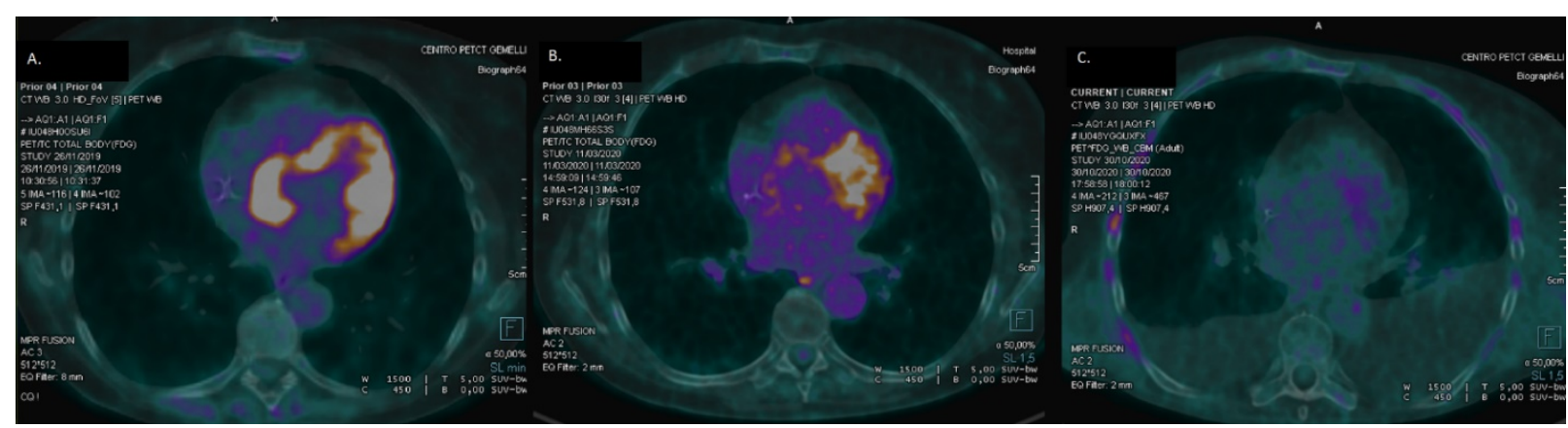

Figure 3. ${ }^{18} \mathrm{~F}-\mathrm{FDG}$ PET-CT scan: staging $(\mathrm{A})$, one month $(\mathrm{B})$, and eight months $(\mathrm{C})$ after RT reevaluation

The ${ }^{18} \mathrm{~F}$-FDG PET CT scan carried out subsequently on October 2020 confirmed complete cardiac response (see figure 3).

The patient underwent a successful allogeneic hematopoietic stem cell transplantation from an HLA-identical sister in May 2020. At the last follow-up in January 2021, the patient did not report acute or chronic cardiac toxicity signs, and she was considered free from disease (20 months after diagnosis).

Unfortunately, the patient developed mycotic sepsis shortly after and died of systemic transplantation complications in February 2021. 


\section{Discussion}

At present, there are no specific guidelines for PCL treatment. Being a systemic disease, the therapeutic approach must always include $\mathrm{CHT}$. The $\mathrm{R}-\mathrm{CHOP}$ regimen (rituximab + cyclophosphamide, doxorubicin, vincristine, prednisone) currently represents the standard of care for cardiac DLBCL. The role of surgery is still unclear and often limited to a palliative setting or to stabilize the clinical condition. Surgery is usually performed as an emergency procedure for debulking purposes, because of blood flow obstruction. In other cases, it helps the efficacy of $\mathrm{CHT}$, especially when hemodynamics is compromised (1).

The use of RT, alone or as consolidation after $\mathrm{CHT}$, is described in the literature, although it is not considered a standard approach. One of the reasons is related to the risk of acute and late cardiac toxicity, increased by the use of therapeutic regimens containing anthracyclines. CichowskaCwalińska et al. (2) recently published a review on the role of RT in PCL, concluding that, despite the risk of toxicity and an unclear survival benefit, it can be used as a salvage treatment in patients with cardiac $\mathrm{PCL}$ who do not achieve a complete response after $\mathrm{CHT}$.

The goal of using the innovative MRgRT approach was to minimize cardiac toxicity through the use of MR for planning purposes. MR is the preferred imaging modality for cardiac masses due to its best contrast soft-tissue resolution, multiplanar imaging capabilities, and unrestricted field of view (7). This patient's workflow incorporated data from the diagnostic cardiac MR scans to increase contouring accuracy with the 0.35 T TRUFI MR simulation images provided by the hybrid MR Linac. These last images were then used daily for patient positioning and therapy monitoring, according to the usual MRgRT workflow. Furthermore, MR-based gating protocols allow the reduction of uncertainty margins related to therapy volumes, thus avoiding unnecessary irradiation of healthy cardiac surrounding tissue.

\section{Conclusion}

MRgRT is characterized by excellent patient compliance and a favorable toxicity profile and may be considered an option in the multidisciplinary treatment of primary and secondary cardiac lymphoma.

\footnotetext{
Abbreviations:

ASCT - autologous stem cell transplantation 18F-FDG PET-CT - 18F-fluorodeoxyglucose positron emission tomography- computed tomography bSSFP - balanced steady-state free precession.

$\mathrm{CHT}$ - chemotherapy

CT - computed tomography

CTCAE - Common Terminology Criteria for Adverse Events;

$\mathrm{DIBH}$ - deep inspiration breath hold

DLBCL - diffuse large cell B-cell lymphoma

ECG - electrocardiogram

$E F$ - ejection fraction

GMALL-ALL/NHL - German multicenter ALL - acute lymphoblastic leukemia/ Non-Hodgkin's lymphoma

GTV - gross tumor volume

HLA - Human leukocyte antigen

MR - magnetic resonance

MR-Linac - magnetic resonance - linear accelerator

MRgRT - magnetic Resonance guided radiotherapy
} 
MRI - magnetic resonance imaging

OARs - organs at risk

$\mathrm{PCL}$ - primary cardiac lymphoma

R-CHOP - rituximab, cyclophosphamide, doxorubicin, vincristine, prednisone

RECIST - Response evaluation criteria in solid tumors

$\mathrm{RT}$ - radiotherapy

SUV - standard uptake value

TEE - trans esophageal echocardiogram

TRUFI - true fast imaging

TTE - transthoracic echocardiogram

\section{Statements:}

Author's contributions: conceptualization, LB, and AR; methodology, AC and EDM; data curation, MI, FDA and $\mathrm{SH}$; writing — original draft preparation, $\mathrm{LB}$ and $\mathrm{AR}$; writing —review and editing, SC and CM; supervision: MB. All authors have read and agreed to the published version of the manuscript.

Consent for publication: The patient recruited in the study signed an informed consent regarding the publication of their data.

Conflict of interest: Dr. Luca Boldrini has active research agreements with ViewRay Inc (Mountain View, CA, USA) and received speaker honoraria for scientific presentations and travel reimbursements.

Statement of ethics: This research study was conducted retrospectively in accordance with the ethical standards of the institutional and national research committee and with the 1964 Helsinki Declaration and its later amendments or comparable ethical standards.

Ethical approval: Ethics approval was not required for this study

\section{References}

1. Jeudy J, Burke AP, Frazier AA. Cardiac Lymphoma. Radiol. Clin. North Am. 2016;54:689-710.

2. Cichowska-Cwalińska N, Dutka M, Klapkowski A, Pęksa R, Maciej Zaucha J, Zaucha R. The role of radiotherapy in the management of primary cardiac lymphoma a case report and the literature review. Leuk. Lymphoma 2019;60:812-816.

3. Petrich A, Cho SI, Billett $\mathrm{H}$. Primary cardiac lymphoma: an analysis of presentation, treatment, and outcome patterns. Cancer 2011;117:581-589.

4. Jonavicius K, Salcius K, Meskauskas R, Valeviciene N, Tarutis V, Sirvydis V. Primary cardiac lymphoma: two cases and a review of literature. J Cardiothorac Surg 2015;10. Available at: https://www.ncbi.nlm.nih.gov/ pmc/articles/PMC4628386/. Accessed December 3, 2020.

5. Bonou M, Kapelios CJ, Marinakos A, et al. Diagnosis and treatment complications of primary cardiac lymphoma in an immunocompetent 28-year old man: a case report. BMC Cancer 2019;19:191.

6. Klüter S. Technical design and concept of a 0.35 T MR-Linac. Clin Transl Radiat Oncol 2019;18:98-101.

7. Corradini S, Alongi F, Andratschke N, et al. MR-guidance in clinical reality: current treatment challenges and future perspectives. Radiat Oncol 2019;14:92. 